\title{
COFINITENESS OF LOCAL COHOMOLOGY MODULES IN THE CLASS OF MODULES IN DIMENSION LESS THAN A FIXED INTEGER
}

\author{
ALIREZA VAHIDI AND MAHDIEH PAPARI-ZAREI
}

\begin{abstract}
Let $n$ be a non-negative integer, $R$ a commutative Noetherian ring with $\operatorname{dim}(R) \leq n+2, \mathfrak{a}$ an ideal of $R$, and $X$ an arbitrary $R$-module. In this paper, we first prove that $X$ is an $\left(\mathrm{FD}_{<n}, \mathfrak{a}\right)$-cofinite $R$-module if $X$ is an a-torsion $R$-module such that $\operatorname{Hom}_{R}\left(\frac{R}{\mathfrak{a}}, X\right)$ and $\operatorname{Ext}_{R}^{1}\left(\frac{R}{\mathfrak{a}}, X\right)$ are $\mathrm{FD}_{<n}$ $R$-modules. Then, we show that $\mathrm{H}_{\mathfrak{a}}^{i}(X)$ is an $\left(\mathrm{FD}_{<n}, \mathfrak{a}\right)$-cofinite $R$-module and $\left\{\mathfrak{p} \in \operatorname{Ass}_{R}\left(\mathrm{H}_{\mathfrak{a}}^{i}(X)\right): \operatorname{dim}\left(\frac{R}{\mathfrak{p}}\right) \geq n\right\}$ is a finite set for all $i$ when $\operatorname{Ext}_{R}^{i}\left(\frac{R}{\mathfrak{a}}, X\right)$ is an $\mathrm{FD}_{<n} R$-module for all $i \leq n+2$. As a consequence, it follows that $\operatorname{Ass}_{R}\left(\mathrm{H}_{\mathfrak{a}}^{i}(X)\right)$ is a finite set for all $i$ whenever $R$ is a semi-local ring with $\operatorname{dim}(R) \leq 3$ and $X$ is an $\mathrm{FD}_{<1} R$-module. Finally, we observe that the category of $\left(\mathrm{FD}_{<n}, \mathfrak{a}\right)$-cofinite $R$-modules forms an Abelian subcategory of the category of $R$-modules.
\end{abstract}

\section{INTRODUCTION}

We adopt throughout the following notation: let $R$ denote a commutative Noetherian ring with non-zero identity, $\mathfrak{a}$ and $\mathfrak{b}$ ideals of $R, M$ a finite (i.e., finitely generated) $R$-module, $X$ an arbitrary $R$-module which is not necessarily finite, and $n$ a non-negative integer. We refer the reader to [7, 8, 23] for basic results, notations, and terminology not given in this paper.

Hartshorne, in [14, defined an a-torsion $R$-module $X$ to be $\mathfrak{a}$-cofinite if the $R$-module $\operatorname{Ext}_{R}^{i}\left(\frac{R}{\mathfrak{a}}, X\right)$ is finite for all $i$, and asked the following questions.

Question 1.1. Does the category of $\mathfrak{a}$-cofinite $R$-modules form an Abelian subcategory of the category of $R$-modules?

Question 1.2. Is $\mathrm{H}_{\mathfrak{a}}^{i}(M)$ an $\mathfrak{a}$-cofinite $R$-module for all $i$ ?

The following question is also an important problem in local cohomology [16, Problem 4].

Question 1.3. Is $\operatorname{Ass}_{R}\left(\mathrm{H}_{\mathfrak{a}}^{i}(M)\right)$ a finite set for all $i$ ?

2020 Mathematics Subject Classification. 13D07; 13D45.

Key words and phrases. Abelian subcategories; Associated prime ideals; Cofinite modules; Local cohomology modules.

The research of Alireza Vahidi was in part supported by a grant from Payame Noor University. 
There have been many attempts in the literature to study the above questions. Hartshorne in [14, Proposition 7.6 and Corollary 7.7] showed that the answer to these questions is yes if $R$ is a complete regular local ring and $\mathfrak{a}$ is a prime ideal of $R$ with $\operatorname{dim}\left(\frac{R}{a}\right) \leq 1$. Huneke and Koh in [17, Theorem 4.1] and Delfino in [10, Theorem 3] extended Hartshorne's result [14, Corollary 7.7] and provided affirmative answers to Questions 1.2 and 1.3 in more general local rings $R$ and one-dimensional ideals a. Delfino and Marley in [11, Theorems 1 and 2], Yoshida in [25, Theorem 1.1], Chiriacescu in [9, Theorem 1.4], and Kawasaki in [18, Theorems 1 and 8] showed that the answer to Questions $1.1-1.3$ is yes if $R$ is an arbitrary local ring and $\mathfrak{a}$ is an arbitrary ideal of $R$ with $\operatorname{dim}\left(\frac{R}{a}\right) \leq 1$. Finally, in [21, Theorems 7.4 and 7.10] and [22, Theorems 2.6 and 2.10], Melkersson provided affirmative answers to these questions for the case that $R$ is an arbitrary ring and either $\operatorname{dim}(R) \leq 2$ or $\mathfrak{a}$ is an arbitrary ideal of $R$ with $\operatorname{dim}\left(\frac{R}{\mathfrak{a}}\right) \leq 1$.

Recall that $X$ is said to be an $\mathrm{FD}_{<n}$ (or in dimension $<n$ ) $R$-module if there is a finite submodule $Y$ of $X$ such that $\operatorname{dim}_{R}\left(\frac{X}{Y}\right)<n$ [2, 4. From [26, Theorem 2.3], the class of $\mathrm{FD}_{<n} R$-modules is closed under taking submodules, quotients, and extensions. We say that $X$ is an $\left(\mathrm{FD}_{<n}, \mathfrak{a}\right)$-cofinite $R$-module if $X$ is an a-torsion $R$-module and $\operatorname{Ext}_{R}^{i}\left(\frac{R}{\mathfrak{a}}, X\right)$ is an $\mathrm{FD}_{<n} R$-module for all $i$ [3, Definition 4.1]. Note that $X$ is an a-cofinite $R$-module if and only if $X$ is an $\left(\mathrm{FD}_{<0}, \mathfrak{a}\right)$-cofinite $R$-module. Thus, as generalizations of Questions $1.1+1.3$, we have the following questions (see [1, Question] and [24, Questions 1.5, 1.6, and 1.8]). Here, the set $\left\{\mathfrak{p} \in \operatorname{Ass}_{R}(X): \operatorname{dim}\left(\frac{R}{\mathfrak{p}}\right) \geq n\right\}$ is denoted by $\operatorname{Ass}_{R}(X)_{\geq n}$.

Question 1.4. Does the category of $\left(\mathrm{FD}_{<n}, \mathfrak{a}\right)$-cofinite $R$-modules form an Abelian subcategory of the category of $R$-modules?

Question 1.5. Is $\mathrm{H}_{\mathfrak{a}}^{i}(M)$ an $\left(\mathrm{FD}_{<n}, \mathfrak{a}\right)$-cofinite $R$-module for all $i$ ?

Question 1.6. Is $\operatorname{Ass}_{R}\left(\mathrm{H}_{\mathfrak{a}}^{i}(M)\right)_{\geq n}$ a finite set for all $i$ ?

If $R$ is a complete local ring with $\operatorname{dim}\left(\frac{R}{a}\right) \leq n+1$, then the answer to Questions 1.5 and 1.6 is yes from [1, Theorems 2.5 and 2.10]. In [24, Corollaries 3.3 and 4.5], the first author and Morsali removed the complete local assumption on $R$ and provided affirmative answers to Questions 1.4 1.6 for the case that $\operatorname{dim}\left(\frac{R}{a}\right) \leq n+1$, which are generalizations of Melkersson's results [22, Theorems 2.6 and 2.10]. In this paper, as generalizations of Melkersson's results [21, Theorems 7.4 and 7.10], we show that the answer to Questions 1.41 .6 is also yes if $\operatorname{dim}(R) \leq n+2$. As a consequence, we provide an affirmative answer to Question 1.3 for the case that $R$ is a semi-local ring with $\operatorname{dim}(R) \leq 3$. This result is a generalization of Marley's result in [19] where he showed that the answer to Question 1.3 is yes if $R$ is a local ring with $\operatorname{dim}(R) \leq 3$ (see [19, Proposition 1.1 and Corollary 2.5]).

In the main result of Section 2, we observe that if $\operatorname{dim}(R) \leq n+2$ and $X$ is an $\mathfrak{a}$-torsion $R$-module such that $\operatorname{Hom}_{R}\left(\frac{R}{\mathfrak{a}}, X\right)$ and $\operatorname{Ext}_{R}^{1}\left(\frac{R}{\mathfrak{a}}, X\right)$ are $\mathrm{FD}_{<n}$ $R$-modules, then $X$ is an $\left(\mathrm{FD}_{<n}, \mathfrak{a}\right)$-cofinite $R$-module. Section 3 is devoted to the study of Questions 1.5 and 1.6 We show that $\mathrm{H}_{\mathfrak{a}}^{i}(X)$ is an $\left(\mathrm{FD}_{<n}, \mathfrak{a}\right)$-cofinite $R$-module and $\operatorname{Ass}_{R}\left(\mathrm{H}_{\mathfrak{a}}^{i}(X)\right)_{\geq n}$ is a finite set for all $i$ whenever $\operatorname{dim}(R) \leq n+2$ and $\operatorname{Ext}_{R}^{i}\left(\frac{R}{\mathfrak{a}}, X\right)$ is an $\mathrm{FD}_{<n} R$-module for all $i \leq n+2$ (e.g., $X$ is an $\mathrm{FD}_{<n} R$-module). 
It follows that if $R$ is a semi-local ring with $\operatorname{dim}(R) \leq 3$ and $\operatorname{Ext}_{R}^{i}\left(\frac{R}{\mathfrak{a}}, X\right)$ is an $\mathrm{FD}_{<1} R$-module for all $i \leq 3$ (e.g., $X$ is an $\mathrm{FD}_{<1} R$-module), then $\mathrm{H}_{\mathfrak{a}}^{i}(X)$ is an $\mathfrak{a}$ weakly cofinite $R$-module and $\operatorname{Ass}_{R}\left(\mathrm{H}_{\mathfrak{a}}^{i}(X)\right)$ is a finite set for all $i$. Recall that $X$ is said to be an a-weakly cofinite $R$-module if $X$ is an $\mathfrak{a}$-torsion $R$-module and the set of associated prime ideals of any quotient module of $\operatorname{Ext}_{R}^{i}\left(\frac{R}{\mathfrak{a}}, X\right)$ is finite for all $i$ (see [12, Definition 2.1] and [13, Definition 2.4]). In Section 4, with respect to Question 1.4 , we prove that when $\operatorname{dim}(R) \leq n+2$, the category of $\left(\mathrm{FD}_{<n}, \mathfrak{a}\right)$-cofinite $R$-modules forms an Abelian subcategory of the category of $R$-modules.

\section{A CRITERION FOR COFINITENESS}

The following two lemmas will be useful in the proof of the main result of this section. Note that when $\mathfrak{b} X=0, X$ is an $\mathrm{FD}_{<n} R$-module if and only if $X$ is an $\mathrm{FD}_{<n} \frac{R}{\mathfrak{b}}$-module.

Lemma 2.1. Let $t$ be a non-negative integer and let $X$ be an $R$-module such that $\mathfrak{b} X=0$ and $\operatorname{Ext}_{R}^{i}\left(\frac{R}{\mathfrak{a}+\mathfrak{b}}, X\right)$ is an $\mathrm{FD}_{<n} R$-module for all $i \leq t$. Then $\operatorname{Ext}_{\frac{R}{\mathfrak{b}}}^{i}\left(\frac{R}{\mathfrak{a}+\mathfrak{b}}, X\right)$ is an $\mathrm{FD}_{<n} \frac{R}{\mathfrak{b}}$-module for all $i \leq t$.

Proof. We prove this by using induction on $t$. The case $t=0$ is clear from the isomorphisms

$$
\operatorname{Hom}_{\frac{R}{\mathfrak{b}}}\left(\frac{R}{\mathfrak{a}+\mathfrak{b}}, X\right) \cong\left(0:_{X} \frac{\mathfrak{a}+\mathfrak{b}}{\mathfrak{b}}\right) \cong\left(0:_{X} \mathfrak{a}+\mathfrak{b}\right) \cong \operatorname{Hom}_{R}\left(\frac{R}{\mathfrak{a}+\mathfrak{b}}, X\right) .
$$

Suppose that $t>0$ and that $t-1$ is settled. It is enough to show that $\operatorname{Ext}_{\frac{R}{\mathfrak{b}}}^{t}\left(\frac{R}{\mathfrak{a}+\mathfrak{b}}, X\right)$ is an $\mathrm{FD}_{<n} \frac{R}{\mathfrak{b}}$-module, since $\operatorname{Ext}_{\frac{R}{\mathfrak{b}}}^{i}\left(\frac{R}{\mathfrak{a}+\mathfrak{b}}, X\right)$ is an $\mathrm{FD}_{<n} \frac{R}{\mathfrak{b}}$-module for all $i \leq t-1$ by the induction hypothesis on $t-1$. From [23, Theorem 11.65], there is a spectral sequence

$$
\mathrm{E}_{2}^{p, q}:=\operatorname{Ext}_{\frac{R}{\mathfrak{b}}}^{p}\left(\operatorname{Tor}_{q}^{R}\left(\frac{R}{\mathfrak{b}}, \frac{R}{\mathfrak{a}+\mathfrak{b}}\right), X\right) \Longrightarrow \operatorname{Ext}_{R}^{p+q}\left(\frac{R}{\mathfrak{a}+\mathfrak{b}}, X\right) .
$$

Let $r \geq 2$ and set $B_{r}^{t, 0}:=\operatorname{Im}\left(\mathrm{E}_{r}^{t-r, r-1} \longrightarrow \mathrm{E}_{r}^{t, 0}\right)$. Then $B_{r}^{t, 0}$ is an $\mathrm{FD}_{<n} \frac{R}{\mathfrak{b}}$-module because $\mathrm{E}_{r}^{t-r, r-1}$ is a subquotient of $\mathrm{E}_{2}^{t-r, r-1}$ that is an $\mathrm{FD}_{<n} \frac{R}{\mathfrak{b}}$-module by the induction hypothesis and [15, Proposition 3.4]. Thus, from the short exact sequence

$$
0 \longrightarrow B_{r}^{t, 0} \longrightarrow \mathrm{E}_{r}^{t, 0} \longrightarrow \mathrm{E}_{r+1}^{t, 0} \longrightarrow 0,
$$

$\mathrm{E}_{r}^{t, 0}$ is an $\mathrm{FD}_{<n} \frac{R}{\mathfrak{b}}$-module whenever $\mathrm{E}_{r+1}^{t, 0}$ is an $\mathrm{FD}_{<n} \frac{R}{\mathfrak{b}}$-module. There exists a finite filtration

$$
0=\phi^{t+1} H^{t} \subseteq \phi^{t} H^{t} \subseteq \cdots \subseteq \phi^{1} H^{t} \subseteq \phi^{0} H^{t}=\operatorname{Ext}_{R}^{t}\left(\frac{R}{\mathfrak{a}+\mathfrak{b}}, X\right)
$$

such that $\mathrm{E}_{\infty}^{t-i, i} \cong \frac{\phi^{t-i} H^{t}}{\phi^{t-i+1} H^{t}}$ for all $i, 0 \leq i \leq t$. By assumption, $\operatorname{Ext}_{R}^{t}\left(\frac{R}{\mathfrak{a}+\mathfrak{b}}, X\right)$ is an $R$-module. Thus, as we noted at the beginning of this section, $\operatorname{Ext}_{R}^{t}\left(\frac{R}{\mathfrak{a}+\mathfrak{b}}, X\right)$ is an $\mathrm{FD}_{<n} \frac{R}{\mathfrak{b}}$-module and hence $\phi^{t} H^{t}$ is an $\mathrm{FD}_{<n} \frac{R}{\mathfrak{b}}$-module. Therefore $\mathrm{E}_{\infty}^{t, 0} \cong \frac{\phi^{t} H^{t}}{\phi^{t+1} H^{t}}$ is an $\mathrm{FD}_{<n} \frac{R}{\mathfrak{b}}$-module and so $\mathrm{E}_{t+2}^{t, 0}$ is an $\mathrm{FD}_{<n} \frac{R}{\mathfrak{b}}$-module, because $\mathrm{E}_{\infty}^{t, 0}=\mathrm{E}_{t+2}^{t, 0}$ as 
$\mathrm{E}_{j}^{t-j, j-1}=0=\mathrm{E}_{j}^{t+j, 1-j}$ for all $j \geq t+2$. Thus $\mathrm{E}_{2}^{t, 0}=\operatorname{Ext}_{\frac{R}{\mathfrak{b}}}^{t}\left(\frac{R}{\mathfrak{a}+\mathfrak{b}}, X\right)$ is an $\mathrm{FD}_{<n}$ $\frac{R}{\mathfrak{b}}$-module.

Lemma 2.2. Let $t$ be a non-negative integer and let $X$ be an $R$-module such that $\mathfrak{b} X=0$ and $\operatorname{Ext}_{\frac{R}{\mathfrak{b}}}^{i}\left(\frac{R}{\mathfrak{a}+\mathfrak{b}}, X\right)$ is an $\mathrm{FD}_{<n} \frac{R}{\mathfrak{b}}$-module for all $i \leq t$. Then $\operatorname{Ext}_{R}^{i}\left(\frac{R}{\mathfrak{a}}, X\right)$ is an $\mathrm{FD}_{<n} R$-module for all $i \leq t$.

Proof. From [23, Theorem 11.65], there is a spectral sequence

$$
\mathrm{E}_{2}^{p, q}:=\operatorname{Ext}_{\frac{R}{\mathfrak{b}}}^{p}\left(\operatorname{Tor}_{q}^{R}\left(\frac{R}{\mathfrak{b}}, \frac{R}{\mathfrak{a}}\right), X\right) \underset{p}{\Longrightarrow} \operatorname{Ext}_{R}^{p+q}\left(\frac{R}{\mathfrak{a}}, X\right) .
$$

Let $0 \leq j \leq i \leq t$. By [15, Proposition 3.4], $\mathrm{E}_{2}^{i-j, j}$ is an $\mathrm{FD}_{<n} \frac{R}{\mathfrak{b}}$-module. Hence $\mathrm{E}_{\infty}^{i-j, j}$ is an $\mathrm{FD}_{<n} \frac{R}{\mathfrak{b}}$-module as $\mathrm{E}_{\infty}^{i-j, j}=\mathrm{E}_{i+2}^{i-j, j}$ and $\mathrm{E}_{i+2}^{i-j, j}$ is a subquotient of $\mathrm{E}_{2}^{i-j, j}$. There exists a finite filtration

$$
0=\phi^{i+1} H^{i} \subseteq \phi^{i} H^{i} \subseteq \cdots \subseteq \phi^{1} H^{i} \subseteq \phi^{0} H^{i}=\operatorname{Ext}_{R}^{i}\left(\frac{R}{\mathfrak{a}}, X\right)
$$

such that $\mathrm{E}_{\infty}^{i-j, j} \cong \frac{\phi^{i-j} H^{i}}{\phi^{i-j+1} H^{i}}$ for all $j, 0 \leq j \leq i$. Now, from the short exact sequences

$$
0 \longrightarrow \phi^{i-j+1} H^{i} \longrightarrow \phi^{i-j} H^{i} \longrightarrow \mathrm{E}_{\infty}^{i-j, j} \longrightarrow 0,
$$

for all $j, 0 \leq j \leq i, \operatorname{Ext}_{R}^{i}\left(\frac{R}{\mathfrak{a}}, X\right)$ is an $\mathrm{FD}_{<n} \frac{R}{\mathfrak{b}}$-module. Therefore $\operatorname{Ext}_{R}^{i}\left(\frac{R}{\mathfrak{a}}, X\right)$ is an $\mathrm{FD}_{<n} R$-module.

We are now ready to state and prove the main result of this section, which plays an important role in Sections 3 and 4 to study Questions 1.41 .6 .

Theorem 2.3. Suppose that $\operatorname{dim}(R) \leq n+2$ and $X$ is an a-torsion $R$-module such that $\operatorname{Hom}_{R}\left(\frac{R}{\mathfrak{a}}, X\right)$ and $\operatorname{Ext}_{R}^{1}\left(\frac{R}{\mathfrak{a}}, X\right)$ are $\mathrm{FD}_{<n} R$-modules. Then $X$ is an $\left(\mathrm{FD}_{<n}, \mathfrak{a}\right)$-cofinite $R$-module.

Proof. Assume that $\mathfrak{a}$ is nilpotent. Then $\mathfrak{a}^{t}=0$ for some integer $t$. By [15, Proposition 3.4], $\operatorname{Hom}_{R}\left(\frac{R}{\mathfrak{a}^{t}}, X\right)$ is an $\mathrm{FD}_{<n} R$-module and hence $X=\left(0: X \mathfrak{a}^{t}\right)$ is an $\left(\mathrm{FD}_{<n}, \mathfrak{a}\right)$-cofinite $R$-module. Now, assume that $\mathfrak{a}$ is not nilpotent. Since $\Gamma_{\mathfrak{a}}(R)$ is finite, there is an integer $t$ such that $\left(0:_{R} \mathfrak{a}^{t}\right)=\Gamma_{\mathfrak{a}}(R)$. Set $\mathfrak{b}:=\left(0:_{R} \mathfrak{a}^{t}\right)$ and $Y:=\frac{X}{\left(0: \mathfrak{X}^{t}\right)}$. It is easy to see that $\mathfrak{b} Y=0, Y$ is an $(\mathfrak{a}+\mathfrak{b})$-torsion $R$-module, and $\operatorname{dim}\left(\frac{R}{\mathfrak{a}+\mathfrak{b}}\right) \leq n+1$. Since $\left(0:_{X} \mathfrak{a}^{t}\right), \operatorname{Hom}_{R}\left(\frac{R}{\mathfrak{a}+\mathfrak{b}}, X\right)$, and $\operatorname{Ext}_{R}^{1}\left(\frac{R}{\mathfrak{a}+\mathfrak{b}}, X\right)$ are $\mathrm{FD}_{<n} R$-modules from [15, Proposition 3.4], $\operatorname{Hom}_{R}\left(\frac{R}{\mathfrak{a}+\mathfrak{b}}, Y\right)$ and $\operatorname{Ext}_{R}^{1}\left(\frac{R}{\mathfrak{a}+\mathfrak{b}}, Y\right)$ are $\mathrm{FD}_{<n} R$-modules by the short exact sequence

$$
0 \longrightarrow\left(0: X \mathfrak{a}^{t}\right) \longrightarrow X \longrightarrow Y \longrightarrow 0 .
$$

Thus, from [24, Corollary 2.3], $\operatorname{Ext}_{R}^{i}\left(\frac{R}{\mathfrak{a}+\mathfrak{b}}, Y\right)$ is an $\mathrm{FD}_{<n} R$-module for all $i$. Hence $\operatorname{Ext}_{R}^{i}\left(\frac{R}{\mathfrak{a}}, Y\right)$ is an $\mathrm{FD}_{<n} R$-module for all $i$ by Lemmas 2.1 and 2.2 Therefore $X$ is an $\left(\mathrm{FD}_{<n}, \mathfrak{a}\right)$-cofinite $R$-module from the above short exact sequence.

The following corollary is an immediate application of the above theorem. 
Corollary 2.4. Suppose that $\operatorname{dim}(R) \leq n+2$ and $X$ is an arbitrary $R$-module such that $\operatorname{Hom}_{R}\left(\frac{R}{\mathfrak{a}}, X\right)$ and $\operatorname{Ext}_{R}^{1}\left(\frac{R}{\mathfrak{a}}, X\right)$ are $\mathrm{FD}_{<n} R$-modules. Then $\Gamma_{\mathfrak{a}}(X)$ is an $\left(\mathrm{FD}_{<n}, \mathfrak{a}\right)$-cofinite $R$-module.

Proof. By the short exact sequence

$$
0 \longrightarrow \Gamma_{\mathfrak{a}}(X) \longrightarrow X \longrightarrow \frac{X}{\Gamma_{\mathfrak{a}}(X)} \longrightarrow 0,
$$

$\operatorname{Hom}_{R}\left(\frac{R}{\mathfrak{a}}, \Gamma_{\mathfrak{a}}(X)\right)$ and $\operatorname{Ext}_{R}^{1}\left(\frac{R}{\mathfrak{a}}, \Gamma_{\mathfrak{a}}(X)\right)$ are $\mathrm{FD}_{<n} R$-modules. Thus the assertion follows from Theorem 2.3

By putting $n=0$ in Theorem 2.3 and Corollary 2.4 we have the following results.

Corollary 2.5. Suppose that $\operatorname{dim}(R) \leq 2$ and $X$ is an $\mathfrak{a}$-torsion $R$-module such that $\operatorname{Hom}_{R}\left(\frac{R}{\mathfrak{a}}, X\right)$ and $\operatorname{Ext}_{R}^{1}\left(\frac{R}{\mathfrak{a}}, X\right)$ are finite $R$-modules. Then $X$ is an $\mathfrak{a}$-cofinite $R$-module.

Corollary 2.6. Suppose that $\operatorname{dim}(R) \leq 2$ and $X$ is an arbitrary $R$-module such that $\operatorname{Hom}_{R}\left(\frac{R}{\mathfrak{a}}, X\right)$ and $\operatorname{Ext}_{R}^{1}\left(\frac{R}{\mathfrak{a}}, X\right)$ are finite $R$-modules. Then $\Gamma_{\mathfrak{a}}(X)$ is an $\mathfrak{a}$-cofinite $R$-module.

\section{Cofiniteness AND ASSOCiATED PRIMES of LOCAL COHOMOlOGY MOdUles}

The following is the main result of this section; it shows that the answer to Questions 1.5 and 1.6 is yes if $\operatorname{dim}(R) \leq n+2$.

Theorem 3.1. Suppose that $\operatorname{dim}(R) \leq n+2$ and $X$ is an arbitrary $R$-module. Then the following statements are equivalent:

(i) $\mathrm{H}_{\mathfrak{a}}^{i}(X)$ is an $\left(\mathrm{FD}_{<n}, \mathfrak{a}\right)$-cofinite $R$-module for all $i$;

(ii) $\operatorname{Ext}_{R}^{i}\left(\frac{R}{\mathfrak{a}}, X\right)$ is an $\mathrm{FD}_{<n} R$-module for all $i$;

(iii) $\operatorname{Ext}_{R}^{i}\left(\frac{R}{\mathfrak{a}}, X\right)$ is an $\mathrm{FD}_{<n} R$-module for all $i \leq n+2$.

Proof. (i) $\Rightarrow$ (ii). This follows by [3, Theorem 2.1].

(iii) $\Rightarrow(\mathrm{i})$. We first show that if $t$ is a non-negative integer such that $\operatorname{Ext}_{R}^{i}\left(\frac{R}{\mathfrak{a}}, X\right)$ is an $\mathrm{FD}_{<n} R$-module for all $i \leq t+1$, then $\mathrm{H}_{\mathfrak{a}}^{i}(X)$ is an $\left(\mathrm{FD}_{<n}, \mathfrak{a}\right)$-cofinite $R$-module for all $i \leq t$. We prove this by using induction on $t$. The case $t=0$ follows from Corollary 2.4 Suppose that $t>0$ and that $t-1$ is settled. It is enough to show that $\mathrm{H}_{\mathfrak{a}}^{t}(X)$ is an $\left(\mathrm{FD}_{<n}, \mathfrak{a}\right)$-cofinite $R$-module, because $\mathrm{H}_{\mathfrak{a}}^{i}(X)$ is an $\left(\mathrm{FD}_{<n}, \mathfrak{a}\right)$-cofinite $R$-module for all $i \leq t-1$ from the induction hypothesis on $t-1$. By [3, Theorem 2.3], $\operatorname{Hom}_{R}\left(\frac{R}{\mathfrak{a}}, \mathrm{H}_{\mathfrak{a}}^{t}(X)\right)$ and $\operatorname{Ext}_{R}^{1}\left(\frac{R}{\mathfrak{a}}, \mathrm{H}_{\mathfrak{a}}^{t}(X)\right)$ are $\mathrm{FD}_{<n} R$-modules. Therefore $\mathrm{H}_{\mathfrak{a}}^{t}(X)$ is an $\left(\mathrm{FD}_{<n}, \mathfrak{a}\right)$-cofinite $R$-module from Theorem 2.3 This terminates the induction argument. Thus $\mathrm{H}_{\mathfrak{a}}^{i}(X)$ is an $\left(\mathrm{FD}_{<n}, \mathfrak{a}\right)$-cofinite $R$-module for all $i \neq n+2$ from [7, Theorem 6.1.2]. By [3, Theorem 2.3], $\operatorname{Hom}_{R}\left(\frac{R}{\mathfrak{a}}, \mathrm{H}_{\mathfrak{a}}^{n+2}(X)\right)$ is an $\mathrm{FD}_{<n}$ $R$-module. Also, from [7, Exercise 7.1.7], $\operatorname{Supp}_{R}\left(\mathrm{H}_{\mathfrak{a}}^{n+2}(X)\right) \subseteq \operatorname{Max}(R)$, because each $R$-module can be viewed as the direct limit of its finite submodules. Thus $\mathrm{H}_{\mathfrak{a}}^{n+2}(X)$ is an $\left(\mathrm{FD}_{<n}, \mathfrak{a}\right)$-cofinite $R$-module by [24, Lemma 2.1]. 
Corollary 3.2. Suppose that $\operatorname{dim}(R) \leq n+2, X$ is an arbitrary $R$-module, and $t$ is a non-negative integer such that $\operatorname{Ext}_{R}^{i}\left(\frac{R}{a}, X\right)$ is an $\mathrm{FD}_{<n} R$-module for all $i \leq t+1$ (resp. for all $i \leq n+2)$. Then $\mathrm{H}_{\mathfrak{a}}^{i}(X)$ is an $\left(\mathrm{FD}_{<n}, \mathfrak{a}\right)$-cofinite $R$-module for all $i \leq t$ (resp. for all $i)$. In particular, $\operatorname{Ass}_{R}\left(\mathrm{H}_{\mathfrak{a}}^{i}(X)\right)_{\geq n}$ is a finite set for all $i \leq t$ (resp. for all $i)$.

Proof. The first assertion follows from the proof of Theorem 3.1. The last assertion follows by the first one and [8, Exercise 1.2.28].

We have the following corollaries by taking $n=0$ in Theorem 3.1 and Corollary 3.2

Corollary 3.3 (see [21, Theorem 7.10]). Suppose that $\operatorname{dim}(R) \leq 2$ and $X$ is an arbitrary $R$-module. Then the following statements are equivalent:

(i) $\mathrm{H}_{\mathfrak{a}}^{i}(X)$ is an $\mathfrak{a}$-cofinite $R$-module for all $i$;

(ii) $\operatorname{Ext}_{R}^{i}\left(\frac{R}{\mathfrak{a}}, X\right)$ is a finite $R$-module for all $i$;

(iii) $\operatorname{Ext}_{R}^{i}\left(\frac{R}{\mathfrak{a}}, X\right)$ is a finite $R$-module for all $i \leq 2$.

Corollary 3.4. Suppose that $\operatorname{dim}(R) \leq 2$ and $X$ is an arbitrary $R$-module such that $\operatorname{Ext}_{R}^{i}\left(\frac{R}{\mathfrak{a}}, X\right)$ is a finite $R$-module for all $i \leq 2$. Then $\operatorname{Ass}_{R}\left(\mathrm{H}_{\mathfrak{a}}^{i}(X)\right)$ is a finite set for all $i$.

If $R$ is a local ring with $\operatorname{dim}\left(\frac{R}{\mathfrak{a}}\right) \leq 2$, then the answer to Question 1.3 is yes by Bahmanpour-Naghipour's result [6, Theorem 3.1] (see also [20, Theorem 3.3(c)]). In [24, Corollary 5.6], the first author and Morsali generalized this result to arbitrary semi-local rings. In the next result, by putting $n=1$ in Corollary 3.2 we provide an affirmative answer to Question 1.3 for the case that $R$ is a semi-local ring with $\operatorname{dim}(R) \leq 3$. Note that our result is a generalization of Marley's result in [19], where he showed that if $R$ is a local $\operatorname{ring}$ with $\operatorname{dim}(R) \leq 3$ and $M$ is a finite $R$-module, then $\operatorname{Ass}_{R}\left(\mathrm{H}_{\mathfrak{a}}^{i}(M)\right)$ is a finite set for all $i$ (see [19, Proposition 1.1 and Corollary 2.5]). Note also that, if $R$ is a semi-local ring and $X$ is an $\left(\mathrm{FD}_{<1}, \mathfrak{a}\right)$ cofinite $R$-module, then $X$ is an a-weakly cofinite $R$-module by [5, Theorem 3.3].

Corollary 3.5. Suppose that $R$ is a semi-local ring with $\operatorname{dim}(R) \leq 3, X$ is an arbitrary $R$-module, and $t$ is a non-negative integer such that $\operatorname{Ext}_{R}^{i}\left(\frac{R}{a}, X\right)$ is an $\mathrm{FD}_{<1} R$-module for all $i \leq t+1$ (resp. for all $\left.i \leq 3\right)$. Then $\mathrm{H}_{\mathfrak{a}}^{i}(X)$ is an $\mathfrak{a}$-weakly cofinite $R$-module for all $i \leq t$ (resp. for all $i$ ). In particular, $\operatorname{Ass}_{R}\left(\mathrm{H}_{\mathfrak{a}}^{i}(X)\right)$ is a finite set for all $i \leq t$ (resp. for all $i)$.

\section{Abelianness of the CAtegory of Cofinite modules}

The following theorem is the main result of this section; it shows that the answer to Question 1.4 is also yes if $\operatorname{dim}(R) \leq n+2$.

Theorem 4.1. If $\operatorname{dim}(R) \leq n+2$, then the category of $\left(\mathrm{FD}_{<n}, \mathfrak{a}\right)$-cofinite $R$-modules forms an Abelian subcategory of the category of $R$-modules. 
Proof. The proof is similar to that of [24, Theorem 3.1]. We bring it here for the sake of completeness. Assume that $X$ and $Y$ are $\left(\mathrm{FD}_{<n}, \mathfrak{a}\right)$-cofinite $R$-modules and $f: X \rightarrow Y$ is an $R$-homomorphism. We show that $\operatorname{ker} f, \operatorname{im} f$, and coker $f$ are $\left(\mathrm{FD}_{<n}, \mathfrak{a}\right)$-cofinite $R$-modules. From the short exact sequence

$$
0 \longrightarrow \operatorname{im} f \longrightarrow Y \longrightarrow \operatorname{coker} f \longrightarrow 0
$$

$\operatorname{Hom}_{R}\left(\frac{R}{\mathfrak{a}}, \operatorname{im} f\right)$ is an $\mathrm{FD}_{<n} R$-module. Hence $\operatorname{Hom}_{R}\left(\frac{R}{\mathfrak{a}}, \operatorname{ker} f\right)$ and $\operatorname{Ext}_{R}^{1}\left(\frac{R}{\mathfrak{a}}, \operatorname{ker} f\right)$ are $\mathrm{FD}_{<n} R$-modules by the short exact sequence

$$
0 \longrightarrow \operatorname{ker} f \longrightarrow X \longrightarrow \operatorname{im} f \longrightarrow 0 \text {. }
$$

Therefore ker $f$ is an $\left(\mathrm{FD}_{<n}, \mathfrak{a}\right)$-cofinite $R$-module by Theorem 2.3 . Thus im $f$ and coker $f$ are $\left(\mathrm{FD}_{<n}, \mathfrak{a}\right)$-cofinite $R$-modules from the above short exact sequences.

As an immediate application of the above theorem, we have the following corollary.

Corollary 4.2. Suppose that $\operatorname{dim}(R) \leq n+2, N$ is a finite $R$-module, and $X$ is an $\left(\mathrm{FD}_{<n}, \mathfrak{a}\right)$-cofinite $R$-module. Then $\operatorname{Ext}_{R}^{j}(N, X)$ and $\operatorname{Tor}_{j}^{R}(N, X)$ are $\left(\mathrm{FD}_{<n}, \mathfrak{a}\right)$ cofinite $R$-modules for all $j$.

We have the following results by taking $n=0$ in Theorem 4.1 and Corollary 4.2

Corollary 4.3 (see [21, Theorem 7.4]). If $\operatorname{dim}(R) \leq 2$, then the category of $\mathfrak{a}$-cofinite $R$-modules forms an Abelian subcategory of the category of R-modules.

Corollary 4.4. Suppose that $\operatorname{dim}(R) \leq 2, N$ is a finite $R$-module, and $X$ is an $\mathfrak{a}$-cofinite $R$-module. Then $\operatorname{Ext}_{R}^{j}(N, X)$ and $\operatorname{Tor}_{j}^{R}(N, X)$ are a-cofinite $R$-modules for all $j$.

\section{ACKNOWLEDGEMENTS}

The authors would like to thank the referee for the invaluable comments on the manuscript.

\section{REFERENCES}

[1] N. Abazari and K. Bahmanpour, Extension functors of local cohomology modules and Serre categories of modules, Taiwanese J. Math. 19 (2015), no. 1, 211-220. MR 3313413

[2] M. Aghapournahr and K. Bahmanpour, Cofiniteness of weakly Laskerian local cohomology modules, Bull. Math. Soc. Sci. Math. Roumanie (N.S.) 57(105) (2014), no. 4, 347-356. MR 3288929

[3] M. Aghapournahr, A. J. Taherizadeh and A. Vahidi, Extension functors of local cohomology modules, Bull. Iranian Math. Soc. 37 (2011), no. 3, 117-134. MR 2901589

[4] D. Asadollahi and R. Naghipour, Faltings' local-global principle for the finiteness of local cohomology modules, Comm. Algebra 43 (2015), no. 3, 953-958. MR 3298115

[5] K. Bahmanpour, On the category of weakly Laskerian cofinite modules, Math. Scand. 115 (2014), no. 1, 62-68. MR 3250048

[6] K. Bahmanpour and R. Naghipour, Cofiniteness of local cohomology modules for ideals of small dimension, J. Algebra 321 (2009), no. 7, 1997-2011. MR 2494753

[7] M. P. Brodmann and R. Y. Sharp, Local Cohomology: An Algebraic Introduction with Geometric Applications, Cambridge Studies in Advanced Mathematics, 60, Cambridge University Press, Cambridge, 1998. MR 1613627 
[8] W. Bruns and J. Herzog, Cohen-Macaulay rings, Cambridge Studies in Advanced Mathematics, 39, Cambridge University Press, Cambridge, 1993. MR 1251956

[9] G. Chiriacescu, Cofiniteness of local cohomology modules over regular local rings, Bull. London Math. Soc. 32 (2000), no. 1, 1-7. MR 1718769

[10] D. Delfino, On the cofiniteness of local cohomology modules, Math. Proc. Cambridge Philos. Soc. 115 (1994), no. 1, 79-84. MR 1253283

[11] D. Delfino and T. Marley, Cofinite modules and local cohomology, J. Pure Appl. Algebra 121 (1997), no. 1, 45-52. MR 1471123

[12] K. Divaani-Aazar and A. Mafi, Associated primes of local cohomology modules, Proc. Amer. Math. Soc. 133 (2005), no. 3, 655-660. MR 2113911

[13] K. Divaani-Aazar and A. Mafi, Associated primes of local cohomology modules of weakly Laskerian modules, Comm. Algebra 34 (2006), no. 2, 681-690. MR 2211948

[14] R. Hartshorne, Affine duality and cofiniteness, Invent. Math. 9 (1969/70), 145-164. MR 0257096

[15] S. H. Hassanzadeh and A. Vahidi, On vanishing and cofiniteness of generalized local cohomology modules, Comm. Algebra 37 (2009), no. 7, 2290-2299. MR 2536919

[16] C. Huneke, Problems on local cohomology, in Free resolutions in commutative algebra and algebraic geometry (Sundance, UT, 1990), 93-108, Res. Notes Math., 2, Jones and Bartlett, Boston, MA. MR 1165320.

[17] C. Huneke and J. Koh, Cofiniteness and vanishing of local cohomology modules, Math. Proc. Cambridge Philos. Soc. 110 (1991), no. 3, 421-429. MR 1120477

[18] K. Kawasaki, On a category of cofinite modules which is Abelian, Math. Z. 269 (2011), no. 1-2, 587-608. MR 2836085

[19] T. Marley, The associated primes of local cohomology modules over rings of small dimension, Manuscripta Math. 104 (2001), no. 4, 519-525. MR 1836111.

[20] T. Marley and J. C. Vassilev, Cofiniteness and associated primes of local cohomology modules, J. Algebra 256 (2002), no. 1, 180-193. MR 1936885

[21] L. Melkersson, Modules cofinite with respect to an ideal, J. Algebra 285 (2005), no. 2, 649-668. MR 2125457

[22] L. Melkersson, Cofiniteness with respect to ideals of dimension one, J. Algebra 372 (2012), 459-462. MR 2990020

[23] J. J. Rotman, An Introduction to Homological Algebra, Pure and Applied Mathematics, 85, Academic Press, New York, 1979. MR 0538169

[24] A. Vahidi and S. Morsali, Cofiniteness with respect to the class of modules in dimension less than a fixed integer, Taiwanese J. Math. 24 (2020), no. 4, 825-840. MR 4124548.

[25] K.-I. Yoshida, Cofiniteness of local cohomology modules for ideals of dimension one, Nagoya Math. J. 147 (1997), 179-191. MR 1475172.

[26] T. Yoshizawa, Subcategories of extension modules by Serre subcategories, Proc. Amer. Math. Soc. 140 (2012), no. 7, 2293-2305. MR 2898693

\author{
Alireza Vahidi ${ }^{凶}$ \\ Department of Mathematics, Payame Noor University (PNU), P.O. Box 19395-4697 Tehran, Iran \\ vahidi.ar@pnu.ac.ir \\ Mahdieh Papari-Zarei \\ Department of Mathematics, Payame Noor University (PNU), P.O. Box 19395-4697 Tehran, Iran \\ m.p.zarei@gmail.com
}

Received: October 15, 2019

Accepted: March 19, 2020 